\title{
TOWARDS ROBOT COMPANIONS
}

\author{
Raja Chatila
}

LAAS-CNRS, 7 av du Colonel Roche, 31077 TOULOUSE Cedex 4, France

We address the challenges of developing a new generation of robots that would be acting along with humans in their daily life and sharing their space.

In order to interact efficiently with humans in changing situations and to be able to accomplish various tasks, such robots should exhibit cognitive capacities for environment understanding, situation awareness and assessment, learning, and decision-making.

The talk will discuss the concepts and approaches for achieving these capacities. The robot being initially endowed with an elementary set of sensor data processings and motor actuation, it builds sensory-motor representations by associating perceptions with actions. This process is based on distributed multiscale representations that are incrementally built through a reinforcement learning mechanism. Situations, defined as associations of spatial layouts and temporal sequences are learned in the same manner. The robot behavior is driven by the need to increase a value function. 